\title{
PROPUESTA CONCEPTUAL DE UN MODELO DE GESTIÓN DEL CONOCIMIENTO PARA UNA UNIVERSIDAD PÚBLICA: CASO FACULTAD DE INGENIERÍA UNIVERSIDAD DE CÓRDOBA.
}

\author{
CONCEPTUAL PROPOSAL OF A MODEL OF KNOWLEDGE MANAGEMENT FOR \\ PUBLIC UNIVERSITY: CASE SCHOOL OF ENGINEERING UNIVERSITY OF CORDOBA
}

\author{
Oswaldo Tordecilla Díaz ${ }^{1}$ \\ Recibido para publicación: 11 de noviembre 2014- Aceptado para publicación: 21 de noviembre 2014
}

\begin{abstract}
RESUMEN
El presente trabajo ha sido direccionado a concebir una propuesta conceptual de un Modelo de Gestión del Conocimiento para una universidad pública "Caso Facultad de Ingeniería: Universidad de Córdoba", soportándose en un diagnostico que identificó la situación real de la facultad en cuanto a un modelo de gestión del conocimiento formalmente establecido. El modelo en su contexto, debe contribuir a optimizar el uso y socialización del conocimiento en la facultad de ingeniería, con el fin de impactar los procesos misionales de Investigación, Docencia, Extensión, Gestión Organizacional y el entorno local y nacional, tomando como referente el modelo SECI2 de creación de conocimiento.
\end{abstract}

Palabras clave: Modelo Gestión del Conocimiento, SECI, Universidad de Córdoba, Facultad de Ingeniería, Gestión, Optimizar, Socialización

\begin{abstract}
This work has been directed to devise a proposal for a Conceptual Model of Knowledge Management for a public university "Case School of Engineering University of Córdoba", supporting a diagnosis that identified the real situation of the faculty as a model knowledge management formally established. The model in context, should help to optimize the use and socialization of knowledge in the engineering faculty, in order to impact the mission processes of research, teaching, extension, Organizational Management and the local and national environment taking as reference the SECI model of knowledge creation .
\end{abstract}

\footnotetext{
${ }_{1}^{1}$ Oswaldo Tordecilla Díaz, Universidad de Córdoba Montería, Colombia (E-mail: oswaldotordecilla@unicordoba.edu.co)

2 http://www.12manage.com/methods_nonaka_seci_es.html
} 


\section{INTRODUCCIÓN}

En una sociedad avocada a estar soportada en el conocimiento, las universidades en su contexto general, están llamadas a ser un actor fundamental en la generación de nuevas ideas a través de la dinámica o interacción constante entre los procesos misionales de la docencia, investigación y extensión. El proceso de investigación debe trascender desde lo básico a la aplicación del nuevo conocimiento en el ámbito sobre el cual debe brindar solución a una problemática planteada a través del desarrollo de un nuevo producto, servicio o proceso, preferiblemente articulando la triada Universidad - Empresa - Estado9, aspirando a impactar con calidad a través de la innovación. Para alcanzar estos ideales desde la perspectiva universitaria, se evidencia la necesidad de implementar en estos centros del saber, estrategias académicas y organizacionales que toquen las fronteras del conocimiento y trasciendan. En consecuencia las universidades a pesar de ser generadoras de nuevo saber, no deben ser ajenas a disciplinas como la Gestión del Conocimiento que debe ser un pilar esencial que dinamice y potencie la producción científica en una espiral de continuo crecimiento, en un entorno global, procurando alcanzar siempre la excelencia para extender o llevar a la sociedad, soluciones a través de productos con altos estándares de calidad. Este ejercicio de investigación es un recorrido al interior de la facultad de ingeniería y por ende al de la universidad de córdoba, que tiene la intensión de denotar y connotar los procesos de la gestión del conocimiento a una facultad que en hipótesis puede presentar carencias al respecto, es así que se aborda un estudio cualitativo y descriptivo, que proporcione las evidencias necesarias para la sustentación de una idea fundamentada en soportes e instrumentos de investigación, que permiten precisar y sostener argumentos lógicos coherentes, y no proponer un doxa sin sentido.

\footnotetext{
${ }^{9} \mathrm{http}: / /$ www.universidadempresaestado.com.co/index.php ?option=com_content\&view=article\&id=8\&Itemid=4
}

\section{ESTADO DEL ARTE}

Teniendo en cuenta que el siglo XXI se caracteriza por ser la era del conocimiento, en donde los activos intangibles [1] pueden ser clasificados como activos o competencias. Los intangibles como activos se refieren a derechos de propiedad intelectual como las patentes, marcas o ciertas tecnologías de la información como las bases de datos que pueden o no estar protegidas legalmente, y los activos como competencias se refieren a los conocimientos de los empleados, clientes o proveedores, los cuales son recursos más difíciles de retener puesto depende de las personas, en tal sentido La Gestión del Conocimiento se ha convertido en una disciplina de gran valor, teniendo en cuenta que debería contribuir en forma directa a apoyar la estrategia organizacional, cuando desde la Gestión del Conocimiento se aporta conocimiento pertinente para la toma de decisiones, tanto en el ámbito académico como empresarial. Gestionar el Conocimiento viene a ser la gestión de todos los activos intangibles (marcas, patentes, licencias, procesos, experiencias, ideas y conocimientos) que aportan valor a la organización a la hora de conseguir capacidades, o competencias esenciales distintivas, que por lo regular aportan ventajas competitivas.

Pero ¿qué es formalmente?, existen muchas interpretaciones y modelos, entre los cuales podemos referenciar los siguientes:

Es la capacidad de una organización para crear nuevo conocimiento, diseminarlo a través de la organización y expresarlo en productos, servicios y sistemas [2].

Es la tarea de reconocer un activo humano enterrado en las mentes de las personas y convertirlo en activo empresarial al que puedan acceder y que pueda ser utilizado por un mayor número de personas [3].

Es el proceso Sistemático de encontrar, seleccionar, organizar, destilar y presentar la información de una manera que mejore la comprensión de un área específica de interés para los miembros de una organización [4].

Es la necesidad de acelerar el flujo de la información que tiene valor, desde los 
individuos a la organización y de vuelta a los individuos, de modo que ellos puedan usarla para crear valor para los clientes [5].

Es la función que planifica, coordina y controla los flujos de conocimiento que se producen en la organización en relación con sus actividades y con su entorno con el fin de crear unas competencias esenciales [6].

Es un proceso organizacional y estratégico de carácter sistemático y sistémico, que busca integrar los procesos de adquisición, captura, creación, asimilación, transferencia y aplicación del conocimiento en la organización, apoyándose en la gestión de la información, la tecnología, el aprendizaje desde el talento humano con el propósito de formar capacidades dinámicas desde una cultura de innovación que sean fuentes de ventajas competitivas, fuentes de creación de valor para los stakeholder y la sociedad en

General [7].

No es un producto de software, ni tampoco una categoría de software. No es ni siquiera una cuestión de técnica. Es algo que empieza con los objetivos y los procesos de la empresa y con el reconocimiento de la necesidad de compartir información. La gestión del conocimiento no es más que gestionar los flujos de la información y llevar la correcta a las personas que la necesitan de manera que sea posible hacer algo con prontitud [8].

Es un conjunto de procesos que permiten utilizar el conocimiento como factor clave para añadir y generar valor [9].

En cuanto a referentes de trabajos de Modelos de Gestión del Conocimiento internacionales y nacionales, citamos a continuación entre muchos los siguientes:

\section{Contexto Internacional}

Universidad del País Vasco: Este trabajo de la universidad del país vasco (UPV/EHU) presenta la manera como se afronta la gestión del conocimiento científico técnico, en esta institución educativa, para la búsqueda de un modelo de gestión, que tiene como propósito la organización eficiente de la producción científica y técnica producida por la universidad. Este proyecto fue la primera parte de un amplio programa que pretende diseñar, implementar y evaluar un nuevo modelo de gestión de conocimiento que permita en un futuro, gestionar modelos para otros procesos tales como docencia y administración.

\section{Contexto Nacional.}

KM: Gestión del Conocimiento Universidad de Pamplona Herramienta de la Universidad de Pamplona (Colombia) que permite recopilar, organizar, analizar y compartir el conocimiento, producido por los investigadores de la Universidad, poniéndolo a disposición de otras instituciones, centros de investigación y desarrollo, grupos de investigación, gremios, incubadoras de empresas, parques tecnológicos, tanques de pensamiento y personas, con la finalidad de consolidar una comunidad de conocimiento a nivel nacional e internacional e igualmente [10] expresa que las universidades de Antioquia han sido particularmente exitosas en su esfuerzo por crear una base organizativa sólida para su investigación, aportando al país el $17 \%$ de los grupos de investigación reconocidos y el $30 \%$ de los grupos de máxima categoría". Por otra parte es fundamental traer a colación los pensamiento del autor [11] en cuanto al infinito valor que se le debe dar a los activos intangibles en el sentido de lo práctico y útil para la ventaja competitiva de una empresa, hoy por hoy una empresa debe aprovechar al máximo las experiencias, a los gestores del conocimiento que pueden llegar a conocer a los empleados y pueden llegar a saber quiénes tienen un especial conocimiento sobre un tema particular, aprovechando el mismo a favor de la organización.

Cuando el tamaño y dispersión hacen imposible el conocimiento directo de quién es

quién. ¿Cómo acceder al experto? ¿Cómo encontrar lo que se necesita? El conocimiento es valioso en la medida en que sea accesible, con el fin de evitar reinventar constantemente la rueda, la duplicación de esfuerzos, otro caso ejemplo en Colombia es el Modelo para el desarrollo de la Gestión del Conocimiento en los Centros de Investigación de las Universidades Públicas Colombianas. Caso Aplicativo Universidad Pedagógica y Tecnológica de Colombia (UPTC) Este trabajo se realiza como respuesta al deterioro de los procesos de investigación en la UPTC, la falta de políticas institucionales encargadas de gestionar e impulsar la investigación basadas en la gestión del conocimiento y la necesidad de incentivar y 
mejorar la producción científica y académica de los centros y grupos de investigación.

El estudio, en su parte final, reveló que el tiempo de existencia y los integrantes, específicamente los docentes de planta y los jóvenes investigadores, son los que de mejor manera ayudan a generar conocimiento. Los grupos de investigación cuentan con capital humano de gran calidad, pero el estímulo es bajo y las condiciones de acceso a la tecnología son limitadas, lo mismo que la recepción de incentivos económicos; por otro lado, se detecta un proceso de tramito-manía para la radicación de las investigaciones, lo que afecta su eficiencia y permite catalogar, en general, a la UPTC en un rango medio de la gestión de conocimiento investigativo. Para finalizar, en la investigación se presentó un modelo teórico, propuestas de desarrollo y consolidación de la gestión del conocimiento en los grupos de la universidad, orientados al mejoramiento continuo y a la creación de lineamientos que permitan identificarlos claramente.

\section{METODOLOGÍA}

El proyecto se desarrolló bajo los preceptos de una investigación cualitativa (descriptiva explicativa), apelando a la técnica del estudio de caso (Facultad de Ingeniería: Universidad de Córdoba).

Para la recolección de información, se utilizó como instrumento la encuesta, la cual se estructuró en módulos temáticos, orientados a obtener una visión holística de la situación real de los elementos de la Gestión del Conocimiento que informalmente se pudieran identificar en la facultad de ingeniería de la universidad de córdoba. La encuesta se aplicó sobre una población de 80 miembros de la comunidad docente y administrativa.

El proceso metodológico se predispuso en seis módulos a saber: Apropiación del Conocimiento Institucional, Transferencia del Conocimiento, Apropiación de Tecnologías, Toma de Decisiones, Seguridad del Conocimiento, Cultura Organizacional, esto con el fin de seleccionar y profundizar en algunas aristas de la Gestión del Conocimiento y extraer información precisa para analizar, relacionar y/o comparar entre ellos, es decir en la medida en que se identifica un componente, se identifican sus fortalezas y debilidades, que al ser comparado con los otros se muestra una panorámica más amplia, que permite posibilitar un estudio holístico, en el sentido de que estudiándolo por separado genera una parcialidad de lo que se quiere, en este orden de ideas un elemento del modelo del conocimiento, permite mirar hacia adentro del alma mater y determinar en este ámbito específico que nos falta en cada uno de los ámbitos referenciados. Fue en este sentido que se realiza una interpretación sobre unos datos arrojados a unas encuestas específicas que permitió hacer una lectura, que se puede decodificar con los siguientes resultados.

\section{RESULTADOS}

El análisis realizado sobre los datos, producto de la aplicación del instrumento (encuesta), muestra interesantes resultados, que alineados con la evidente importancia que para cualquier organización y en especial una Institución de Educación Superior cuya esencia es la producción de conocimiento, debe representar un modelo de Gestión del Conocimiento; dejando entrever fortalezas y debilidades entre elementos inherentes al modelo, tomando como fuente productora de conocimiento los grupos de investigación, la actividad docente y la gestión organizacional en la facultad.

A continuación se referencian los módulos temáticos que estructuraron la encuesta y se muestran algunos resultados:

\section{Apropiación del Conocimiento}

\section{Institucional.}

En qué grado los miembros de la organización tienen interiorizado el cómo, el cuándo y el dónde del quehacer laboral institucional (docencia, investigación, extensión y gestión administrativa), los flujos de información e igualmente la importancia que ello significa para la organización y por supuesto para un modelo de GC.

A continuación se muestran resultados relativos al eje temático. 


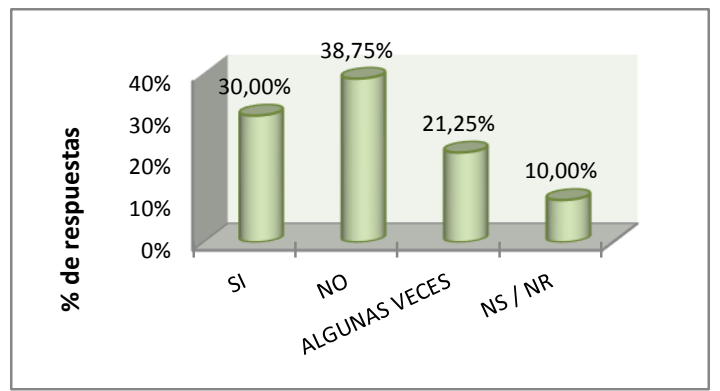

Figura 1. Existencia en la facultad de procedimientos que permitan identificar, codificar y organizar el conocimiento en el área de la investigación

\section{Transferencia del Conocimiento.}

El módulo es orientado a identificar la dinámica de socialización y transferencias de productos y experiencias significativas que se dan alrededor de los ejes misionales y la gestión organizacional en la facultad, teniendo en cuenta que ello debería impactarlos (retroalimentarlos), originando una espiral creciente de creación de conocimiento con estándares de calidad cada vez mayor, lo cual debe tributar en nuevos productos con calidad y en ventajas competitivas que deben convertirse en un factor diferenciador.

Se muestran a continuación algunos resultados significativos sobre los cuales se debe reflexionar.

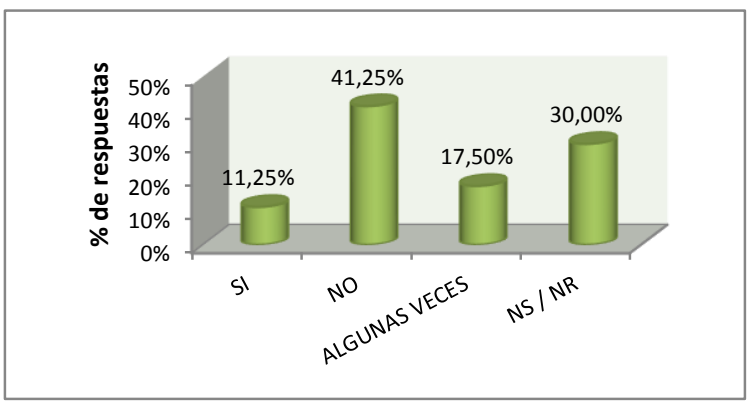

Figura 2. Existen políticas y/o metodologías que garanticen la transferencia de información o conocimientos adquiridos entre los grupos de investigación.

\section{Apropiación de Tecnologías.}

Un Modelo de Gestión del Conocimiento debe estar íntimamente ligado con una fuerte cultura informática y soportado en una sólida infraestructura tecnológica a nivel de herramientas TIC's, que permita a través de una plataforma orientada a la Gestión del
Conocimiento, registrar, codificar, organizar y socializar el mismo, contemplando igualmente herramientas multimediales que soporten la interacción virtual de miembros de comunidades de practica en áreas específicas del saber.

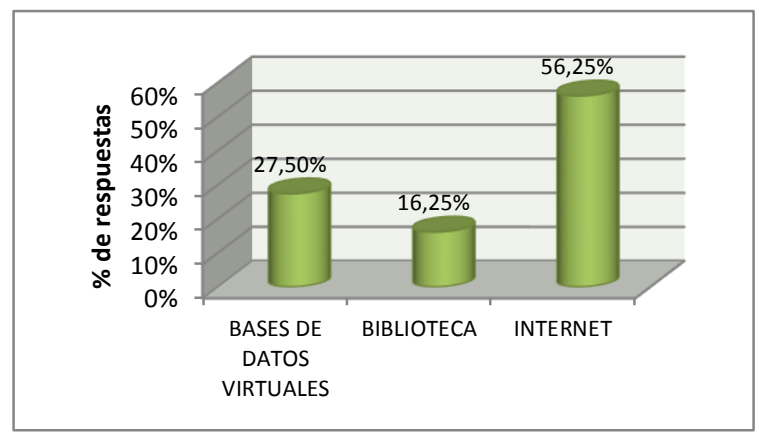

Figura 3. Medios de consulta más usados en el ejercicio de la investigación.

\section{Toma de Decisiones.}

El módulo evidencia la importancia que tiene el tema Toma de Decisiones para cualquier organización y fundamentalmente lo esencial que es contar con un Sistema de Información Orientado a la Toma de Decisiones, del cual los encuestados poseen claridad sobre sus objetivos y necesidad, teniendo en cuenta que actualmente la facultad no cuenta con él, lo cual lógicamente no le permite a directivos hacer un proceso eficiente de verificación, seguimiento, evaluación y retroalimentación a las decisiones tomadas, de lo que se puede inferir que la facultad no cuenta con la sistematización de indicadores de gestión, lo que finalmente es una limitante para tomar decisiones oportunas y eficaces.

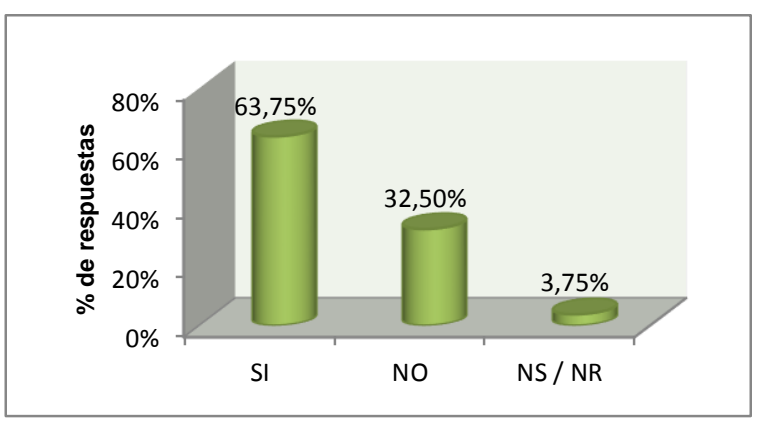

Figura 4. Sistema de Información Orientado a la toma de decisiones. Claridad sobre su importancia. 


\section{Seguridad del Conocimiento.}

El conocimiento entendido como un activo intangible susceptible de ser cuantificado y contabilizado, el cual propicia a la organización ventajas competitivas, requiere ser generado, recolectado, codificado, registrado, procesado, darle valor agregado y ser socializado con la intención de impactar el entorno.

El resultado de la encuesta nos muestra que la facultad requiere de la innovación y la retroalimentación de sus procesos para que todo aquello que sea producto del ejercicio intelectual, este protegido por ella, mediante la gestión y acompañamiento legal para patentar, licenciar y registrar todo lo producido intelectualmente como producto del ejercicio de investigación (básica y aplicada) y los archivos de respaldo deben ser conservados en eficientes bases de datos, almacenadas en servidores institucionales y respaldados mediante sistemas periódicos de backup, que garanticen ante cualquier eventualidad, la recuperación total de la información. Igualmente se evidencia la poca motivación e incentivos para retener todo el conocimiento producido y en particular, aquel que puede pasar de la investigación aplicada a un producto innovador o en otras palabras evitar que lo gestado intelectualmente al interior de la facultad pueda ser objeto de fuga, puesto que su(s) autor(es) pueda $(n)$ recibir mejores propuestas para tal fin por parte de otras instituciones públicas o privadas, lo que tributa en una pérdida del capital intelectual.

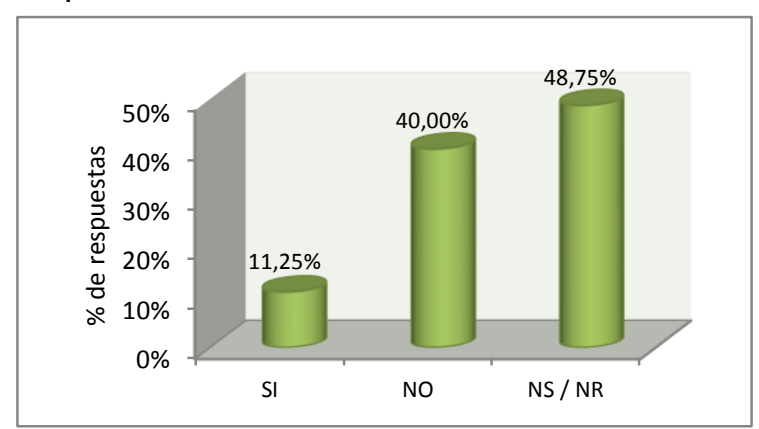

Figura 5. ¿Existe en la Facultad una política de confidencialidad y discrecionalidad, que garantice la no filtración de información?

Propuesta del Modelo Conceptual de Gestión del Conocimiento para la Facultad de Ingeniería de la Universidad de Córdoba. Realizado un análisis riguroso a las encuestas y sopesando el caso de la facultad de ingeniería de la universidad de córdoba, se considera la posibilidad de que esta pueda implementar para sus procesos internos tanto en lo académico como en lo administrativo, incluyendo las proyecciones en cada uno de ellos, un modelo teórico de Gestión del Conocimiento basado en los principios del modelo de Ikujiro Nonaka \& Hirotaka Takeuchi para la creación de conocimiento, el cual esta direccionado hacia la socialización permanente del conocimiento que es precisamente de lo que carece la facultad y la universidad en general. En este sentido el modelo presenta cuatro grandes momentos, el primero de lo tácito a lo tácito, el segundo de lo tácito a lo explicito, el tercero de lo explícito a lo explícito y el cuarto de lo explícito a lo tácito. Ahora bien se entiende por conocimiento tácito "Al tipo de conocimiento personal o propio del individuo, que se basa en modelos mentales, creencias, habilidades individuales y está profundamente vinculado con la experiencia, las emociones y la práctica; lo que dificulta o resulta difícil de comunicarlo a los demás." (Nonaka \& Takeuchi 1995).

La otra parte conocida como conocimiento explicito se identifica de la siguiente manera: "es objetivo y está contenido en manuales, puede ser procesado, transmitido o guardado en bases de datos es fácil de articular y verbalizar, sistemático y objetivo; racional y lógico, digital, secuencial y del pasado, y libre de contexto. Puede expresarse con un lenguaje formal y sistemático y compartirse en forma de datos, fórmulas científicas, especificaciones 0 manuales (Nonaka \& Takeuchi, 1995). Luego desde esta perspectiva del modelo SECI (Socialización, Externalización, Combinación e Interiorización), se puede concebir como un modelo que encaja perfectamente con las necesidades de la universidad de Córdoba en cuanto a un modelo de la Gestión del Conocimiento, por lo que posibilita la interacción y la externalización del ejercicio y la fluidez del conocimiento dada la razón de ser de la universidad. El modelo SECI no es un modelo estático, más bien es un modelo dinámico que permanece en un constante movimiento, posibilitando el intercambio en todas las direcciones posibles, el cual permite que el conocimiento socializado se fortalezca y se multiplique, muchas veces en un conocimiento nuevo. Ejemplo cuando se habla de tácito a tácito, se puede ofrecer dos posibles lecturas 
para el caso de la facultad (Comunidad Académica). Es aquella situación en la que en un aula de clase el docente transmite una serie de teorías o conceptos que muchas veces reciben retroalimentación por parte de los estudiantes o desde una observación directa o un presaber. La otra lectura puede darse en el campo de lo administrativo cuando un funcionario que ha permanecido en un cargo por un determinado lapso de tiempo, se apropia de un conocimiento dado por la praxis diaria o experiencias, es decir que aprende en el ejercicio laboral.

De lo tácito a lo explícito en el campo de la docencia, se puede considerar cuando cualquier miembro de la comunidad académica, tomando las experiencias u observaciones, es capaz de construir un nuevo conocimiento y registrarlo en repositorios, desde donde posteriormente pueden ser recuperados y consultados. De igual forma sucede en la parte administrativa donde el funcionario puede escribir todas aquellas experiencias que ha acumulado a lo largo de su vida laboral, pasando este conocimiento a ser visible para que otras personas puedan consultar y aprender optimizando el tiempo de aprendizaje, gracias a que fue registrado.

De lo explícito a lo explícito, desde la perspectiva académica se puede considerar aquel ejercicio donde el docente ofrece una teoría X explicitada en un documento, e invita a sus estudiantes que sobre ella realicen una antítesis que rebata los argumentos del autor y utilizando el ensayo como herramienta metodológicxa, que se fundamente en una bibliografía explicita y actualizada. Desde la funcionalidad administrativa cuando un funcionario dadas sus competencias, actualiza, transforma o cambia para mejorar la calidad y los tiempos documentados de los procesos, es decir cuando el funcionario es capaz de ser eficaz.

De lo Explicito a lo tácito cuando aquel miembro de la comunidad académica, habiendo leído un texto extrae un nuevo conocimiento para él, por lo tanto es explicito, ese conocimiento está en él, es el quien lo ha digerido $y$ lo ha interpretado desde su perspectiva, una vez asimilado el conocimiento, lo posterior es transmitirlo a sus discípulos o iguales, pasando en ese momento a un conocimiento tácito, este es el día a día de la universidad. En la parte administrativa el proceso es igual.

Cuando el funcionario socializa y abstrae de normas y/o manuales de procesos, conceptos y los interpreta, amplía a través de sus acertadas experiencias su base de conocimiento, crece él como individuo, y a su vez aprende la universidad en general "organizaciones que aprenden"10, es decir cuando el funcionario competente aprende y socializa, automáticamente aprende y crece también la organización. En este sentido se ha propuesto un modelo del Gestión del Conocimiento, teniendo en cuenta los lineamientos que propone Nonaka \& Takeuchi, que intente en un primer momento engranar todos los actores que interactúan en dirección de un mismo fin y que ese soporte sea la socialización de todos los procesos en una sola plataforma que pueda acopiar, catalogar, registrar, analizar y socializar el conocimiento institucional.

Con fundamento en el resultado del diagnóstico y las posturas planteadas en el marco teórico, específicamente en el modelo SECI de creación de conocimiento, se origina la propuesta de un modelo conceptual para la Facultad de Ingeniería de la Universidad de Córdoba, el cual gráficamente se observa a continuación.

\footnotetext{
${ }^{10}$ Desde esta perspectiva Senge (1994) nos plantea que una organización o empresa en aprendizaje es aquella que "de manera continua y sistemática, se embarca en un proceso para obtener el máximo provecho de sus experiencias aprendiendo de ella" (Núñez, http.//
}

www.degerencia.com). Se basa en la idea de que hay que aprender a ver la realidad con nuevos ojos, detectando ciertas leyes que nos permita entenderla y manejarla. 


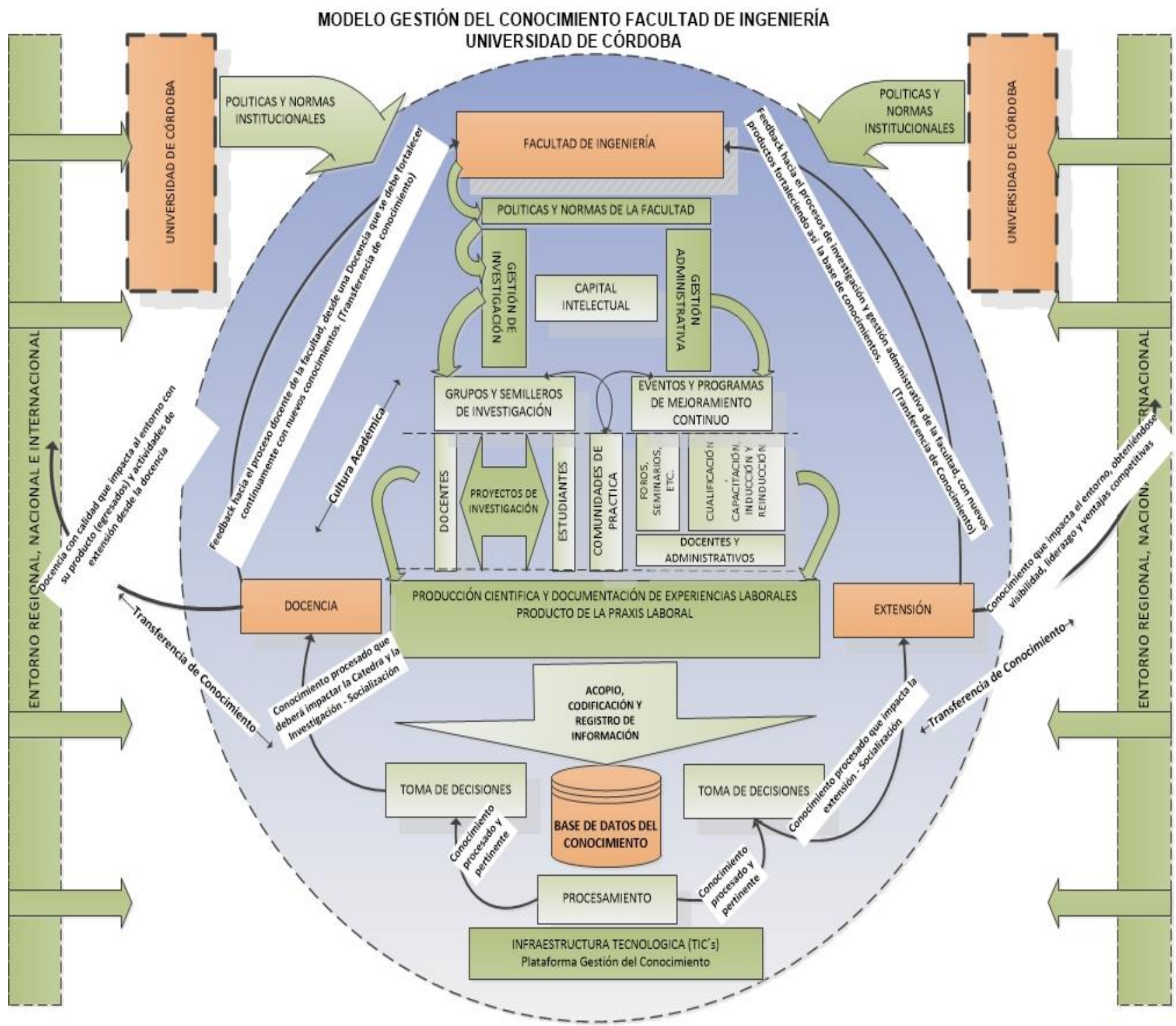

Figura 6. Modelo Conceptual de Gestión del Conocimiento para la Facultad de Ingeniería: Universidad de Córdoba Fuente: Elaboración propia 


\section{CONCLUSIONES}

Desde la perspectiva de este trabajo de investigación, se aporta un diagnostico situacional de la facultad de ingeniería de la Universidad de Córdoba, entorno a la presencia en ella de elementos relativos a la Gestión del Conocimiento, proponiendo al final un Modelo Conceptual de Gestión del Conocimiento ajustado a los preceptos planteados en el Modelo SECl de creación de conocimiento.

Es evidente que en la Facultad se identifica abundante producción de conocimiento, en especial a través del eje misional de la investigación y desde la praxis laboral cotidiana al interactuar con el entorno absorbiendo información que sobre la acción de la combinación y conjugada con las competencias y valores individuales y organizacionales, es convertida en conocimiento, pero es claro que bajo la ausencia de una política direccionada a la Gestión del Conocimiento no solo a nivel de la facultad, sino a nivel general, aspectos importantes como el registro la socialización y transferencia de lo producido no impactan en el grado que se debería al eje misional de la docencia y la misma investigación e igualmente a la gestión organizacional y el entorno. En concordancia se hace palpable la urgente necesidad de contar con una plataforma tecnológica orientada a la Gestión del Conocimiento, que contribuya a optimizar el uso del mismo, garantizando que la información procesada, que luego es convertida en conocimiento circule, proporcionando a los miembros de la Facultad un estado del arte interno que apoye los procesos de investigación, docencia, extensión y nutrirse desde afuera con un Sistema de Vigilancia Tecnológica que monitoree el estado del arte externo en áreas específicas del saber, para aportar a los investigadores, docentes y funcionarios, lo que en la actualidad se está haciendo, quien lo está haciendo y como lo está haciendo.

Efectivamente se pudo observar y analizar el estado en que se encuentra la Facultad de
Ingeniería en este importante tema, posibilitando sostener incluso que la deficiencia en estos procesos de gestión del conocimiento no es un hecho particular de la facultad, sino que permea inclusive a toda la Universidad, encontrando fortalezas y debilidades relacionadas con la Gestión del Conocimiento, citamos entre otras las siguientes:

$\checkmark$ Se evidencia la importancia y el apoyo a la investigación al interior de la facultad.

$\checkmark$ Es poco visible la socialización de la producción investigativa.

$\checkmark$ El trabajo interdisciplinario entre equipos investigativos es deficiente.

$\checkmark$ Se carece de una plataforma que registre, organice y visibilice la producción científica y los resultados de la propedéutica laboral académico - administrativa, no permitiendo de esta manera, que se impacte adecuadamente el ejercicio de la investigación, docencia y extensión.

$\checkmark$ Ausencia de socialización de temáticas tratadas en comunidades de práctica 0 diálogos de saberes.

$\checkmark$ Los procesos académico-administrativos en la facultad no se encuentran documentados en su totalidad, o si lo están, no son socializados adecuadamente $o$ no son de fácil acceso a todos los miembros de la comunidad.

$\checkmark \quad$ Los docentes desconocen cómo actualizar o registrar para la Institución lo que han aprendido.

$\checkmark$ Faltan políticas claras enfocadas a la gestión de patentes, licencias y los beneficios que de ella se puedan desprender para la universidad y el docente.

$\checkmark \quad$ No existen políticas ni metodologías que garanticen la transferencia de información o conocimientos adquiridos entre miembros y grupos de investigación.

$\checkmark \quad$ La Facultad no cuenta con estrategias que permitan retener los conocimientos de los funcionarios que salen de la Institución o son trasladados a otras áreas, no se 
garantiza la conversión del conocimiento tácito a explícito.

$\checkmark$ Se considera importante la rotación del personal como factor que aumenta el conocimiento organizacional, incrementando de esta manera el capital intelectual Institucional, que se ha ido adquiriendo con la experiencia científica y laboral obtenida desde los diferentes ámbitos de trabajo.

$\checkmark \quad$ No existe una plataforma que posibilite hallar todo tipo de información para la investigación, en otras palabras un estado del arte interno.

$\checkmark$ No se aporta valor agregado a la información, para que ella se convierta en un factor diferenciador de la organización y hacerla de esa forma competitiva en el tiempo. Todo lo anterior es factible de alcanzar cuando a parte de la tecnología, se cuenta con el talento humano capacitado para hacer de ella una constante aliada y no un obstáculo o excusa para no_avanzar en los proyectos.

$\checkmark \quad$ Se evidencia la importancia que tiene el tema Toma de Decisiones para cualquier organización y fundamentalmente lo esencial que es contar con un Sistema de Información Orientado a la Toma de Decisiones.

$\checkmark \quad$ Igualmente se evidencia la poca motivación e incentivos para retener todo el conocimiento producido y en particular, aquel que puede pasar de la investigación aplicada a un producto innovador o en otras palabras evitar que lo gestado intelectualmente al interior de la facultad pueda ser objeto de fuga, puesto que su(s) autor(es) pueda(n) recibir mejores propuestas para tal fin por parte de otras instituciones públicas o privadas, lo que tributa en una pérdida del capital intelectual.

De esta forma se puede concluir que el proyecto de investigación alcanzó los objetivos propuestos, arrojando como producto final la propuesta del Modelo Conceptual de Gestión del Conocimiento para la Facultad de Ingeniería de la Universidad de Córdoba e identificando estrategias que deberían tenerse en cuenta para la materialización del modelo como tal.

\section{REFERENCIAS}

[1]. Hall, R., "The Strategic Analysis of Intangible Resources", Strategic Management Journal, 1992, vol. 13.

[2]. Nonaka, I \& Takeuchi, H. (1995). "The Knowledge-Creating company". The Oxford University Press, New York.

[3]. Salazar, C., José M. y Zarandona, A., Xabier (2007). Valoración crítica de los modelos de Gestión del Conocimiento, XXI Congreso Anual AEDEM, Universidad Rey Juan Carlos, Madrid, junio de 2007. Disponible en: http://dialnet.unirioja.es/servlet/libro?codig $0=275423$.

[4]. Pastor Sánchez, J. A. (2000). Gestión del Conocimiento en Instituciones Universitarias. En SCIRE, 6(2), 99-120. Disponible http://ibersid.eu/ojs/index.php/scire/article/v iew/1136/1118.

[5]. Andersen, Arthur (1999) El management en el siglo XXI. Herramientas para los desafíos empresariales de la próxima década. Editorial Granica, Buenos Aires.

[6]. Bueno Campos, E., Morcillo Ortega, P., Rodríguez Pomeda, J., Luque de la Torre, M., Cervera Oliver, M., Camacho Mancilla, C., et. Al. (2003). Gestión del Conocimiento en Universidades y Organismos Públicos de Investigación. Disponible en http://www.madrimasd.org/informacionidi/bi blioteca/publicacion/doc/16 GestionConoc imientoUniversidadesOPIS.pdf.

[7]. Robledo F., Juan Carlos (2009:90). Tesis Doctoral. Gestión del Conocimiento, Teoría y Práctica. Estrategia de Competitividad Empresarial.

[8]. Gates, Bill (1999), Libro - "Los negocios en la era digital".

[9]. Tejedor, B., y Aguirre, A. (1998): "Proyecto Logos: Investigación relativa a la capacidad de aprender de la empresa española", 
Boletín de Estudios Económicos, Vol. LIII, $\mathrm{N}^{\circ}$ 164, Agosto.

[10]. Valhondo, Domingo (2010), Gestión del Conocimiento "Del mito a la Realidad", Ediciones Díaz de Santos, S. A., página 51, Madrid, España. Libro digital.

[11]. Robledo Velásquez, Jorge. (2006). "De los grupos consolidados de Investigación a los Sistemas Dinámicos de Innovación:
El desafío actual del desarrollo científico y tecnológico colombiano", Dyna, julio, año/vol. 74, número 152 Universidad Nacional de Colombia - Medellín Colombia pp. 1-7. Consultado el 20 de mayo de 2012. Disponible en: http://redalyc.uaemex.mx/redalyc/pdf/496 /49615202.pdf. 